Session 1526

\title{
An Optical Communication Design Laboratory
}

\author{
J.A. Buck, H. Owen, J.P. Uyemura, and C.M. Verber \\ School of Electrical and Computer Engineering \\ Georgia Institute of Technology \\ Atlanta, GA 30332
}

D.J. Blumenthal

Department of Electrical Engineering

University of California

Santa Barbara, CA

A senior-level design laboratory course is described, in which an evolving fiber communication network is expanded or improved upon by successive generations of students. In this pipelined approach, the students in each new term base their work on the final written report of the students in the previous term. They choose leaders and organize themselves into teams as appropriate to accomplish the assigned tasks. At the end of the term, they write their own report, which details their work in upgrading the network, and which includes new designs for consideration by the next class. Thus, in addition to its primary goal of providing a high-level technical experience, the course stresses multidisciplinary teamwork, and provides incentive for the development of effective oral and written communication skills. Results of three offerings of the course are described.

\section{INTRODUCTION}

Developing a design laboratory course in optical communication is a formidable challenge, owing to the multidisciplinary nature of the subject. Optics, communications, and electronics expertise at advanced levels are necessary to provide a meaningful design experience for seniors. In addition, equipment and supply costs to establish and maintain such a laboratory are high. The subject course began as a collaboration of faculty members within the required disciplines, who provided expertise and equipment 
from their research laboratories. Initial funding was provided by local industry; subsequent funding came from additional external and internal sources.

A primary objective in establishing the design course was to produce an environment for students that would be similar to what can occur in industry. Specifically, an industrial design team could be given the task of upgrading or improving an existing device or system that was designed by a different team; in the worst case, the previous team no longer exists -- in the sense that none of its members is available to answer questions or provide any verbal guidance. The new team is forced to rely entirely on the written documentation of the previous team, and on the observations and measurements they are able to perform on the existing device or system. On completion of their work, the new team must carefully document their activities for the benefit of future groups.

\section{DESIGN COURSE STRUCTURE}

Our new design course is based on the above scenario, in which an optical fiber communication network is the evolving system. In each course offering, the students are given the final report of the previous student group (most if not all of whom have graduated) and are assigned a general task involving the expansion or improvement of the network. They must understand and evaluate the previous work as documented in the report, so that they can proceed with their assigned task. No other communication with the previous group is allowed. The report also contains suggestions for future activity, including one or more designs for new components or system sub-structures. The new group must evaluate these designs and judge their merit before proceeding. When the new group writes its final report, their own designs for future components or modifications are included, to be evaluated by the next group.

The students choose a leader, organize themselves into sub-groups as necessary, and delegate individual tasks. The faculty member offers initial guidance on procedures, gives technical information when needed, but otherwise plays the role of an active observer, noting progress and individual performance. Lecture time takes the form of round-table group meetings, run by the students, in which the faculty member participates primarily by asking questions. Such questions could typically be of the form, "What are the implications of taking this approach?", or "What measurements would be appropriate to make at this stage?", or "Does this activity make the most efficient use of your team members?". The faculty member will provide advice on request, but the students otherwise make their own decisions.

Students enter the design course (EE4053) with a background consisting of a lecture and a structured laboratory course in fiber communications (courses EE4051 and EE4052). In the structured lab, they are given hands-on training in optical fibers, light sources and detectors, fiber test and measurement equipment, and in methods of evaluating the performance of a basic fiber communication link. In addition to the usual lab book write-ups that they submit for grading, the students are also required to submit two formal written reports during the term. 


\section{THE FIBER COMMUNICATION NETWORK.}

The initial student group's task was to design and construct a 4-subscriber network, capable of supporting data rates of up to $1 \mathrm{GBit} / \mathrm{sec}$, and in which the maximum loss (between the transmitter and receiver of furthest separation) is no greater than $30 \mathrm{~dB}$. They were given several choices of network topologies, including ring, star, and bus geometries. Bus topology was chosen based on its structural simplicity. Specifically, it required the fewest number of splices to construct, and signals do not re-circulate (as in the loop topology), thus requiring special means for their removal. The basic network was constructed by the initial group. Commercial combiners and taps were used to connect the transmitter and receiver modules to the single-mode fiber bus line. Fiber connections were made using mechanical splices, thus allowing the network to be opened at various points for testing. The maximum system loss was within the $30 \mathrm{~dB}$ requirement.

The next student group was given two general tasks: 1) to re-configure the network for synchronous operation by adding clock input taps at the transmitter stations, and 2) to design appropriate drive circuitry for bit frame construction, transmission, and reception, using an elementary SONET-type system protocol. The work included all interface circuitry between the data frame construction logic and the electro-optic transmitter and receiver modules. The interface circuitry problem was in fact the subject of the first offering of the design lab, in which the point-to-point link experiment in the prerequisite fiber lab was improved upon.

The transmitter and receiver units were Laser Diode Inc. models TL-1165 and RT-1554, respectively. The TL-1165 requires either ECL or PECL inputs, and generates optical data at 1.55 micrometers. The RT-1554 detects the optical signal and generates a PECL electronic output. In using these units, it has not been necessary to involve the students with laser drive circuitry, or with the details of receiver design, although this may be necessary at later evolutionary stages. A data rate of $5 \mathrm{OMHz}$ was established as the goal for this stage in the project. The resulting circuitry tested well at $20 \mathrm{MHz}$, but exhibited problems at higher frequencies.

An additional task was to add WDM couplers at appropriate locations to allow twowavelength operation, at 1.3 and 1.55 micrometers. Aside from the obvious attraction of increased channel density by using two wavelengths, this also allowed the possibility of using separate wavelengths for the data and clock pulses in the SONET configuration. Adding the additional taps and WDM couplers increased the maximum loss beyond the original 30dB budget, but using the Laser Diode transmitter and receiver modules increased the allowable loss to over 40dB. The loss of the modified network was found to be within this new limit.

s26Several technical difficulties were encountered, all of which were overcome. In the optical network, students learned that extreme care is needed in avoiding excessive stresses and bends in the over $2 \mathrm{~km}$ of bare fiber segments used in the network construction. Fiber splices were unreliable until methods of performing and stabilizing splices were perfected. The students determined that all mechanical splices would have 
to be replaced by fusion splices once the initial testing was completed. The electronic design problems were generally well-within the capabilities of seniors in electrical engineering. The main problems were in the circuit construction and packaging for low noise operation. As of this writing, the logic circuitry, transmitter and receiver boards, and optical network are constructed, but remain to be joined as a unit. The goal of the next group is to accomplish this, evaluate the performance, and re-design as appropriate.

\section{PERCEIVED BENEFIT AND FUTURE DIRECTIONS}

As of this writing, the design course has been offered four times. We measure success not only by the amount of progress in the network over each term, but by the perceived growth in the students during this time. The most instructive moments in the course in our view occur when crises develop, such as when a mis-measurement has lead to incorrect beliefs on how a particular component behaves, or when a crucial piece of equipment has broken down or is unavailable, thus forcing the team to improvise. Events such as these slow progress enough so that the original objectives may not be met, but we have observed that the learning process at these moments is still of significant value. Student comments in conversations subsequent to the courses have been consistent with these observations. Another benefit to the students is that they are made acutely aware of the value of clear written documentation. It is our experience so far that the final report of each new student group has been of higher quality than those of its predecessors.

The current network will likely expand and improve by increasing its number of subscribers, increasing the data rate, and by increasing its capacity through more advanced WDM implementations. As the sophistication level increases, however, the learning curve for each new group becomes steeper. To accommodate this, additional material on the network is to be incorporated into the prerequisite courses, so that students enter the design course with good familiarity with the network. Another feature that we are now incorporating is optical system simulation software, which will become a design aid for future classes.

\section{CONCLUSION}

We have demonstrated a pipelined approach to a senior level optical communication system design experience, in which successive student groups improve on an existing fiber communication network, having only written documentation of the previous group on which to base their efforts. Three offerings of the course have been successful, in that the network has progressed, and since a learning and maturing process in the student groups has been evident. Students obtain the experience of multidisciplinary teamwork, and establish a strong appreciation for clear written documentation. 